Thorax (1972), 27, 156.

\title{
Pulmonary aspergillomas treated with corticosteroids
}

\author{
DEWI DAVIES and ALAN R. SOMNER
}

\author{
Ransom Hospital, Mansfield and the Chest Clinic, Wallsend-on-Tyne
}

\begin{abstract}
The development of an aspergilloma in a damaged lung is often a silent process, though such patients are more likely to have chronic cough and recurrent haemoptysis.

Two patients are described who had aspergillomas associated with chronic ill health, weight loss, and copious purulent and often bloodstained sputum. Treatment with antibiotics was ineffective but the response to corticosteroids was striking. Reasons are given for believing that the symptoms were due to an Arthus type of antigen-antibody reaction in the cavity wall.
\end{abstract}

When an aspergilloma appears within a cavity in a patient with chronic lung damage the event is usually silent and undramatic. The most common antecedent disease is tuberculosis but aspergillomas may also develop in patients with such conditions as bronchiectasis, sarcoidosis, pneumoconiosis, allergic bronchopulmonary aspergillosis, lung fibrosis associated with ankylosing spondylitis, and idiopathic lung fibrosis (Davies, 1970). Patients with these conditions often have productive cough, breathlessness, and haemoptysis. A large survey has shown that in such patients who also develop an aspergilloma, chronic cough is more likely to be present and the frequency of haemoptysis is increased three to four times (British Thoracic and Tuberculosis Association, 1970). The presence of an aspergilloma, however, does not, in general, have an adverse effect on health or body weight or lead to increased mortality.

The fungus grows on the wall of a cavity and an aspergilloma is formed when mycelium and debris fall off to form a ball. A radiographic change often seen when an aspergilloma develops is that the wall becomes thicker (British Thoracic and Tuberculosis Association, 1970). This is presumably either because of mycelial growth on the wall or because the wall, previously composed of a thin layer of fibrous tissue or epithelium, becomes replaced by thicker granulation tissue. In many cases, however, an epithelial lining is preserved in the presence of an aspergilloma (Kilman, Ahn, Andrews, and Klassen, 1969).

There is general agreement that, despite the frequency of haemoptysis, infiltration and penetration of the cavity wall by the fungus is slight or absent in most cases. Even so, sufficient reaction $\vec{\theta}$ between the fungus and the host occurs to prot duce precipitating antibodies.

An aspergilloma can therefore usually bes regarded as a rather benign complication, no calling for treatment or excision except for actuan or anticipated bleeding or for the elimination of cough and sputum in those patients with localized lung disease.

Occasionally patients with aspergillomas develop? more severe generalized symptoms. Hilvering: Stevens, and Orie (1970) described two patients with aspergillomas who became ill with fever, weight loss, and large amounts of purulent haemorrhagic sputum without evidence of bacterial infection. The first patient improved on corticosteroids in the week before pneumonectomy. The second also improved and was main tained on corticosteroids for two years beforeo dying from respiratory failure. In the first patient the radiograph also showed increasing lungo opacities at the time of clinical deterioration $\bar{N}$ The authors attributed the febrile illness to a types 3 or Arthus antigen-antibody reaction within the lung. As a consequence of finding these patientsu Stevens, Hilvering, and Orie (1970) gave inhala o tions of aspergillus extract to five other patients with aspergilloma. One produced an immediate type 1 bronchial response with dyspnoea, wheez ing, and airways obstruction. Three patients developed a delayed type 3 response witho dyspnoea, malaise, and fever. The remaining one्尺 had combined early and late reactions.

We describe two patients with aspergillomas who have benefited from treatment with corticosteroids. 


\section{CASE REPORTS}

CASE 1 G.F.W., a 52-year-old boiler maker, had been well until 1947. He then began to have attacks of breathlessness, especially at night, with wheezing, cough, and mucoid sputum. He developed nasal polypi and had several operations for their removal between 1947 and 1953. He was regarded as suffering from asthmatic bronchitis and suspected bronchiectasis and in 1956 he developed a left basal pneumonia. In 1959 he was given a pension by his employers as a permanent invalid. At that time he was very thin and had clubbing of the fingers. Bilateral bronchograms showed only minor beading in some of the segmental bronchi. Blood eosinophilia was noted on several occasions and once reached $22 \%$. A considerable polymorph leucocytosis and a high sedimentation rate were frequent findings.

His general health remained very poor, the sputum gradually became more purulent, and he had repeated small haemoptyses.

In 1963 he looked chronically ill, had foul breath, and was producing between 250 and $500 \mathrm{ml}$ of mucopurulent sputum a day. He was afebrile. He had marked clubbing of the fingers and scattered rhonchi and crepitations over both lungs with dullness over the right upper zone. His haemoglobin was $11.0 \mathrm{~g} / 100$ $\mathrm{ml}$, white blood count $13,300 / \mu \mathrm{l}$ with $9 \%$ eosinophils. His sputum showed $20 \%$ eosinophils on one occasion

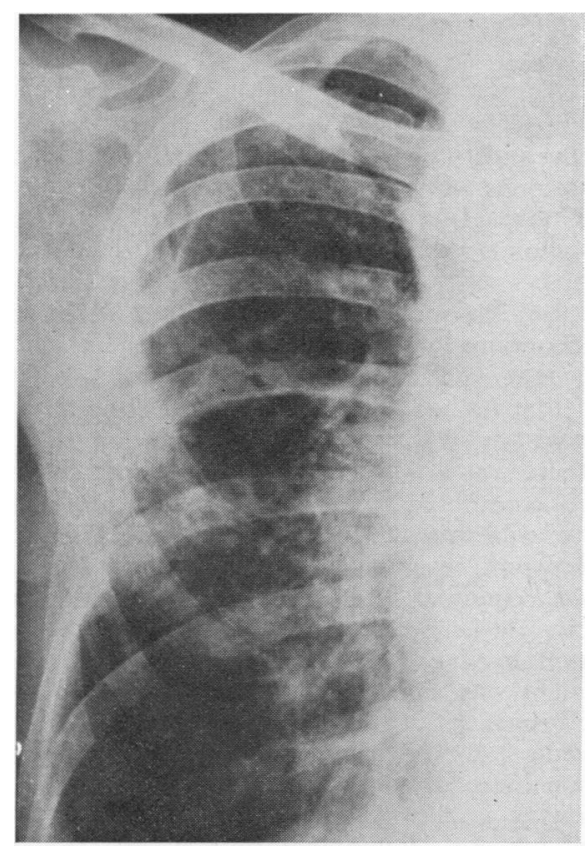

FIG. 1. Case 1. Radiograph in 1960 showing thin-walled cystic spaces in right upper lobe and a general increase in striation. and $5 \%$ on another and cultures produced Haemophilus influenzae and Aspergillus fumigatus on many occasions. Precipitating antibody against $A$. fumigatus was present in the serum. Prick testing gave immediate positive reactions with $A$. fumigatus ( $8 \mathrm{~mm}$ diameter), A. terreus $(5 \mathrm{~mm})$, and grass pollen $(4 \mathrm{~mm})$. Other reactions were negative. His vital capacity was $2 \cdot 15$ litres and the forced expiratory volume in one second $\left(\mathrm{FEV}_{1}\right) \mathbf{1} \cdot 85$ litres.

Apart from pneumonia in 1956 serial chest radiographs from 1948 had been reported to show bronchitic changes or increased basal markings only. The film of February 1960 (Fig. 1), however, showed thin-walled cystic spaces in the right upper lobe and increased striation throughout the rest of the lungs. By 1962 there was more obvious cavitation in the right upper lobe and by April 1963 a large, loosely packed aspergilloma was occupying a very large cavity in the upper half of the right lung.

It was therefore possible to conclude that he had developed asthma in 1947 and that at the same time or later he had been sensitized by $A$. fumigatus. The evidence is insufficient to say whether he had also acquired allergic bronchopulmonary aspergillosis. The cystic changes in the right upper lobe were evidently the consequence of the airways disorder and the cavities then became colonized with $A$. fumigatus.

Treatment with antibiotics and inhalations of brilliant green for one month did not lead to any improvement. He was readmitted to hospital in July 1963 and started on prednisolone, $20 \mathrm{mg}$ daily. Within six days there was a striking reduction in sputum volume and within a month the average daily volume had fallen from $300 \mathrm{ml}$ to $45 \mathrm{ml}$. It remained purulent. He felt much less short of breath, his ventilatory function had improved (Table) and he had

T A B L E

CASE 1: VENTILATORY TESTS AND CORTICOSTEROID DOSAGE

\begin{tabular}{|c|c|c|c|}
\hline & $\begin{array}{c}\text { Vital Capacity } \\
\text { (l.) }\end{array}$ & $\underset{(! .)}{\mathrm{FEV}_{1}}$ & $\begin{array}{l}\text { Prednisolone } \\
\text { (mg/week) }\end{array}$ \\
\hline $\left.\begin{array}{l}\text { July } 1963 \\
\text { After isoprenaline } \\
\text { August } 1963 \\
\text { January } 1964 \\
\text { May } 1966 \\
\text { June } 1970\end{array}\right\}$ & $\begin{array}{l}2 \cdot 25 \\
2 \cdot 70 \\
3 \cdot 05 \\
3 \cdot 00 \\
3 \cdot 10 \\
3 \cdot 15\end{array}$ & $\begin{array}{l}1 \cdot 90 \\
2 \cdot 05 \\
2 \cdot 58 \\
2 \cdot 50 \\
2 \cdot 20 \\
2 \cdot 30\end{array}$ & $\begin{array}{l}\overline{-} \\
\overline{83} \cdot 5 \\
70 \\
50 \\
50\end{array}$ \\
\hline
\end{tabular}

gained $6 \mathrm{~kg}$ in weight. $\mathrm{He}$ continued on prednisolone, $12.5 \mathrm{mg}$ daily. By November 1964 he restarted work after being off since 1959. He has remained on corticosteroids ever since and in recent years has been on prednisolone, $10 \mathrm{mg}$, five days a week. He has occasional absences from work because of 'colds on the chest'. He is short of breath when hurrying and has slight chest tightness. He has scanty purulent sputum which is occasionally bloodstained. Finger clubbing is now very slight. The skin test with $A$. fumigatus extract remains strongly positive and an intradermal 


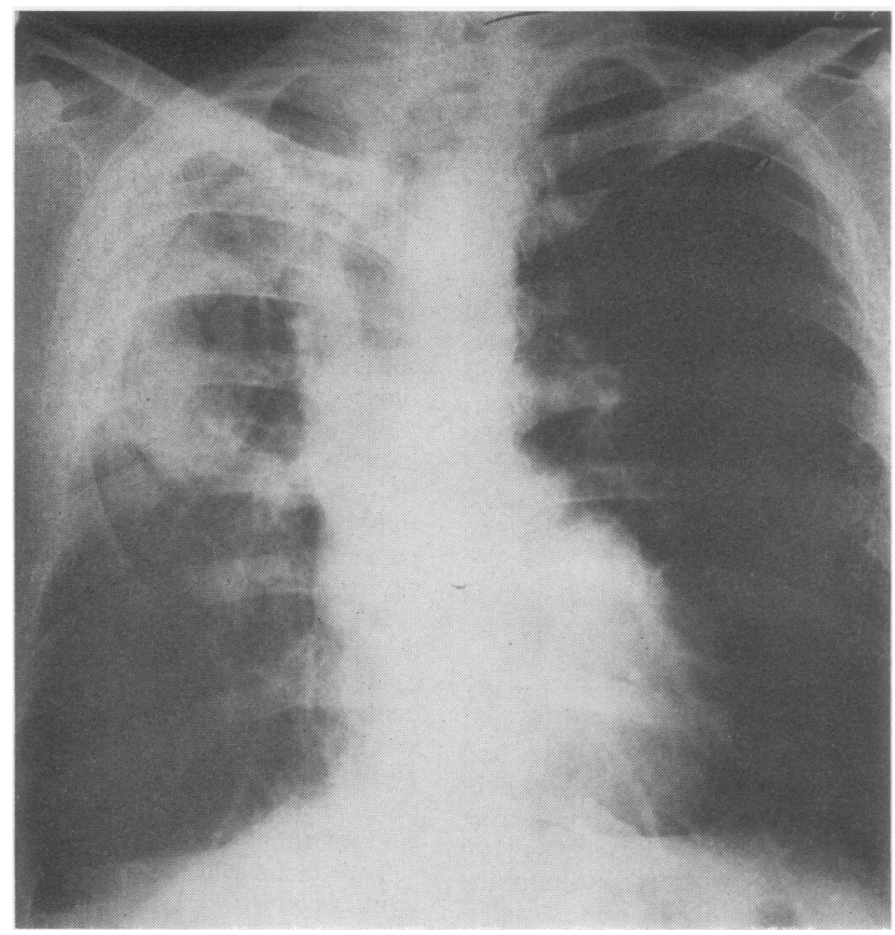

FIG. 2. Case 1. Radiograph in 1969. The cavity in the upper half of the right lung is almost completely filled by the aspergilloma. There is marked overlying pleural thickening.

test does not produce a delayed reaction. The aspergilloma and the cavity have become a little smaller (Fig. 2).

CASE 2 R.W., a 54-year-old man, had a routine chest radiograph in 1953 which was normal apart from evidence of former pleurisy at the left base. In December 1956 he had an haemoptysis. The radiograph then showed patchy shadowing at the left apex (Fig. 3). Sputum tests were negative for Mycobacterium tuberculosis on smear and culture and he was treated with streptomycin, PAS, and isoniazid for four months and with PAS and isoniazid for 13 months. The shadowing at the left apex increased slightly and seemed to be due to pleural thickening.

In March 1959 he had repeated haemoptyses. The radiograph then showed homogenous pleural shadowing at the left apex and diffuse patchy opacities beneath. Sputum tests were negative for Myco. tuberculosis and bronchoscopy showed no abnormality. Streptomycin, PAS, and isoniazid were given for 16 months, during which time haemoptysis occurred on several occasions.

During 1961 and 1962 bleeding recurred at variable intervals and in between his sputum was thick and brown. $A$. fumigatus was repeatedly grown from the sputum and precipitins were found in the serum. Radiographs showed abnormal shadowing in both upper lobes, more marked on the left, where an:aspergilloma was also visible and confirmed by tomography.

In 1963 haemoptysis was almost a daily feature. Bronchograms revealed bronchiectasis in the right upper lobe and some in the left upper lobe. In the belief that he had suppuration of the lung with bron- $\rightarrow$ chiectasis he was given intensive antibiotic therapy‥ and there was a marked reduction in sputum volume. $N$ It was considered that specific treatment for fungus ${ }^{\circ}$ disease was not required. Demethylchlortetracycline 0 (Ledermycin) was given for one year. The sputum N volume remained small and haemoptysis did not re cur. In 1964, three weeks after stopping demethylchlortetracycline, he had a severe haemoptysis an treatment was resumed. He continued to expectorate thick brown sputum.

During 1964 the aspergilloma in the left upper lobe became more obvious and there was an increase of shadowing in the right lung (Figs 4 and 5). He conn tinued to take demethylchlortetracycline and in $1967 \overrightarrow{\mathrm{D}}$ had another brisk haemoptysis. During these yearso he worked fairly regularly and did not complain much. On two occasions the drug was stopped ando 
FIG. 3. Case 2. Radiograph early in 1957 showing patchy shadowing at the left apex and evidence of former pleurisy at left base.
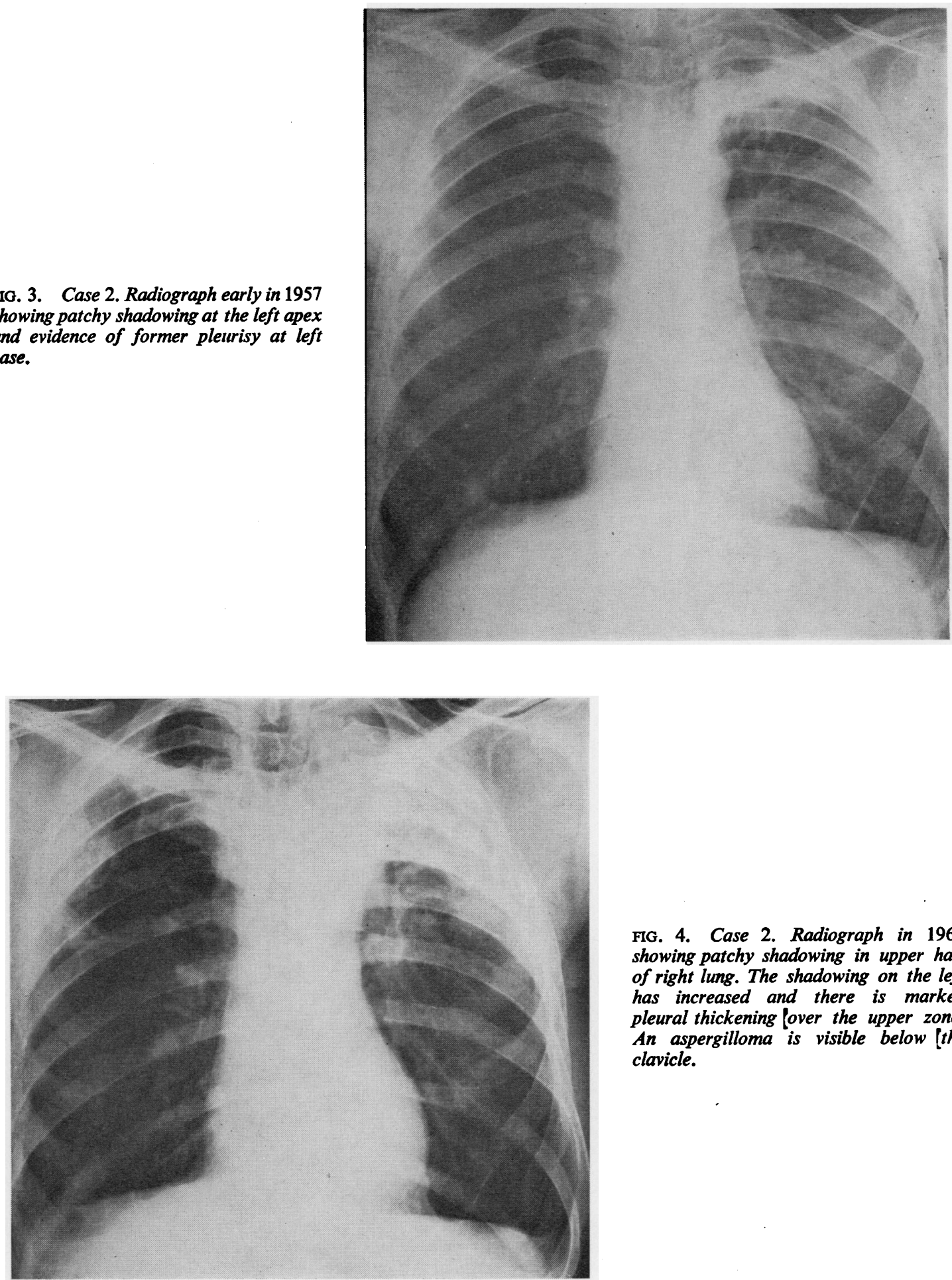

FIG. 4. Case 2. Radiograph in 1964 showing patchy shadowing in upper half of right lung. The shadowing on the left has increased and there is marked pleural thickening lover the upper zone. An aspergilloma is visible below Ithe clavicle. 


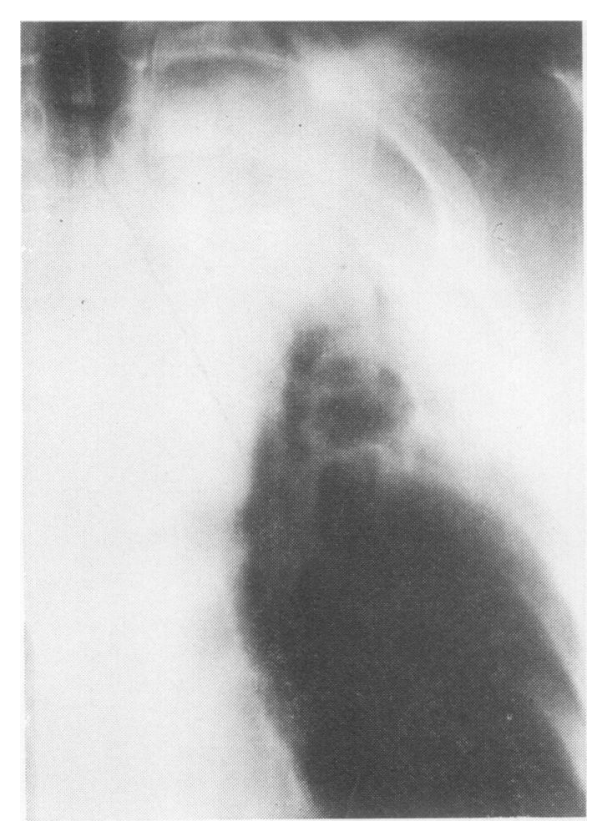

FIG. 5. Case 2. Tomogram of upper part of left lung in 1964 showing an aspergilloma in the upper cavity and an empty cavity below. each time he complained of a considerable increase $\stackrel{0}{x}$ in the volume and bloodstaining of the sputum. How- $\Rightarrow$ ever, in 1969 he began to feel less well, lost weight, $\stackrel{\text { ? }}{+}$ and had a severe cough with plentiful brownisho sputum from which $A$. fumigatus was always isolated. 흐 The chest radiograph showed a considerable increase $\frac{\bar{\sigma}}{}$ of shadowing in the right upper lobe with cavitation $\mathbb{\Phi}$ while, on the left side, cavitation became much more extensive and it appeared that the aspergilloma had कs been coughed up (Fig. 6).

Prednisolone, $20 \mathrm{mg}$ daily, was started on 1 May $\vec{\omega}$ 1970. Four weeks later he reported that it had been o a wonderful month and that he felt 10 years younger. $\vec{x}$ The cough had gone and he had difficulty in pro-i ducing the smallest amount of brownish sputum. In. the second month the sputum became mucoid. The? chest radiograph did not alter.

In July 1970 prednisolone was reduced to $10 \mathrm{mg}$ 우 daily. His general condition then deteriorated and he again had a significant cough and brownish sputum. $\overline{3}$ In November 1970 prednisolone was increased to $15 \frac{\mathrm{N}}{3}$ $\mathrm{mg}$ daily and he quickly experienced striking improve- $\supset$ ment. The skin test with $A$. fumigatus remained $\overrightarrow{0}$ strongly positive and the sputum culture continued to $N$ produce a growth of $A$. fumigatus. The chest radiographs in 1971 showed little change apart from the development of a new aspergilloma on the right.

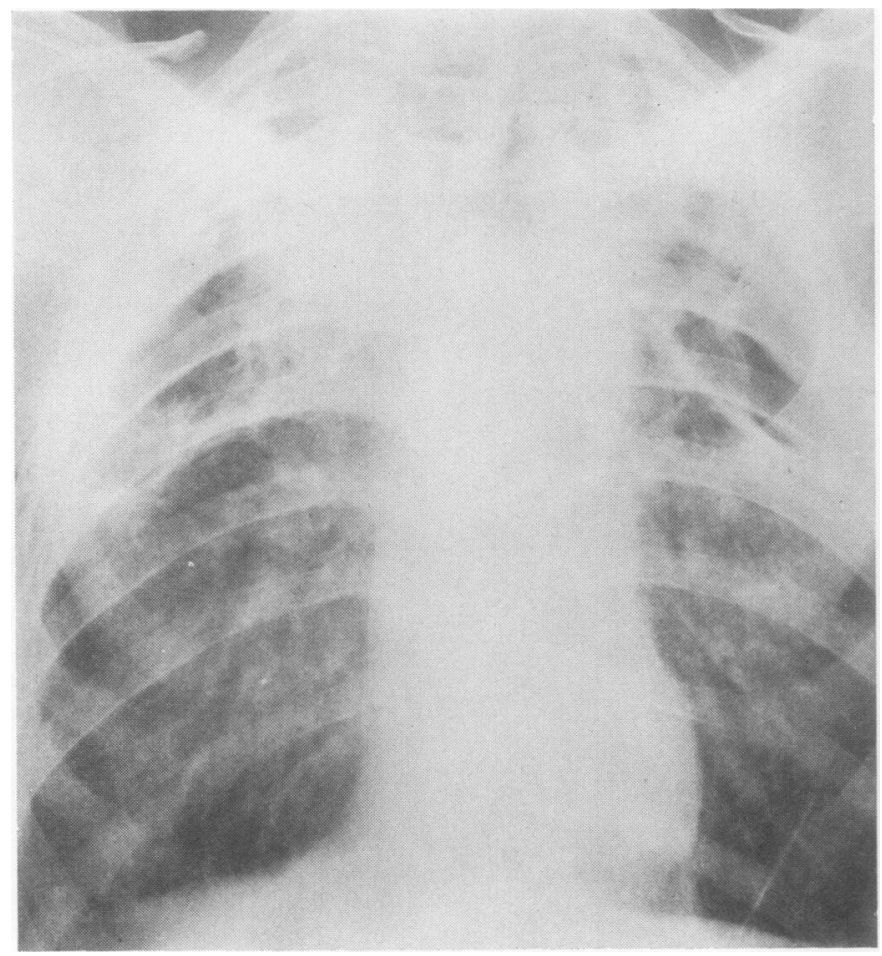

FIG. 6. Case 2. Radiograph in March 1969 showing a marked increase in shadowing and cavitation in right upper lobe. On the left, cavitation is much more extensive and the aspergilloma is no longer visible. 


\section{DISCUSSION}

The first patient had chronic bronchial asthma. It is difficult to be certain whether sensitization to $A$. fumigatus in earlier years had produced only asthma or whether he had developed allergic bronchopulmonary aspergillosis. The characteristic radiographic changes of the latter were not seen nor did he have bronchiectasis (Scadding, 1967) 13 years after the onset of his illness. In allergic bronchopulmonary aspergillosis the clinical symptoms are attributed to a combination of immediate type 1 and delayed type 3 allergy. Both would be suppressed by corticosteroids.

Even if it is assumed that he had allergic bronchopulmonary aspergillosis, the response to corticosteroids was clearly not due to the alleviation of that condition alone. It is a disorder characterized by airways obstruction, episodes of fever, increased dyspnoea, cough, sputum plugs, and segmental or subsegmental shadows on the radiographs. Continuous copious purulent sputum and clubbing of the fingers are not features, except perhaps when severe bronchiectasis has been acquired and this has been complicated by bacterial infection. In such a situation antibiotics would lead to improvement and corticosteroids would be ineffective. The patient had been given antibiotics intermittently for years without benefit.

In the second patient there was no suggestion of asthma or allergic bronchopulmonary aspergillosis, despite the presence of a positive prick test with $\boldsymbol{A}$. fumigatus. Positive reactions are obtainable in about one-third of patients with aspergilloma (British Thoracic and Tuberculosis Association, 1970). Antibiotics had been ineffective.

Since in both patients the reduction in purulent sputum was striking with corticosteroids it seems likely that sputum formation was the result of a type 3 or Arthus antigen-antibody reaction. In such reactions complement-fixing aggregates are formed which are chemotactic for neutrophils and tissue damage may also occur. Malaise and weight loss are characteristic features of a type 3 reaction and were prominent in these patients.

The reasons why such responses are not more common in patients with aspergilloma may be related to the quantitative balance between antigen and antibody. The Arthus reaction occurs when there is moderate antigen excess. Most patients with aspergilloma have strongly positive precipitin tests, indicating that they are well endowed with antibody. Grading of the precipitin test before the administration of corticosteroids had been done in the second patient. He had strong positive reactions in 1964 and 1967 with between five and seven arcs of precipitation on the immuno-electrophoretic test (Longbottom and Pepys, 1964). Both patients may, however, have harboured aspergillomas that were particularly potent in producing large amounts of antigen. The first patient's aspergilloma is the biggest we have seen.

The Arthus reaction is also a feature in allergic bronchopulmonary aspergillosis. Its development is believed to be facilitated by the coexistence of type 1 allergy in such patients. Their precipitin test is usually much less strongly positive than in patients with aspergilloma, indicating that they have less antibody. Though the amount of fungus in the bronchial tree may be relatively small, compared with the amount present in a patient with aspergilloma, sufficient antigen excess seems to be present, particularly during episodes of mucoid impaction of the bronchi (and the trapping of fungus) to produce the reaction. The factors involved in the production of allergic reactions in people infected with aspergilli are considered in greater detail by Pepys (1969).

Some of the clinical differences between patients with allergic bronchopulmonary aspergillosis and the ones we have described may be explained by the location of the Arthus reaction. In the former the reaction is more widespread, even if predominantly in relation to areas of mucoid impaction. It may also be more intermittent, again perhaps dependent on mucoid impaction and the trapping of antigen-producing fungus. In our patients the process was probably confined within the cavity, with more continuous antigen production.

The experiments of Stevens et al. (1970) and our second patient show that type 3 allergy can develop in patients with aspergilloma in the absence of clinical type 1 allergy. The inhalation of large quantities of $\boldsymbol{A}$. clavatus spores in malt workers is known to produce extrinsic allergic alveolitis which is characterized as a type 3 reaction (Riddle et al., 1968). In such patients the antigen is widely distributed at bronchiolar and alveolar levels. We may well have been seeing a comparable antigen-antibody reaction going on within a localized cavity but with the clinical features modified by its confinement.

The second patient also showed progressive lung destruction while on continuous antibiotics. The nature of the underlying disease is not known but it fits into the group called idiopathic lung fibrosis 
(Davies, 1970). It is not clear whether the progressive damage is part of the original disease or whether colonization with aspergillus contributes to this. Such progression is seen in the absence of colonization (Davies, 1970). It is common to find considerable pleural thickening appearing over a cavity when an aspergilloma develops (British Thoracic and Tuberculosis Association, 1970). This may be due to diffusion of toxic material from the cavity or result from an antigen-antibody reaction in the pleura. It would, therefore, not be surprising if colonization of a cavity was sometimes associated with progressive consolidation and fibrosis in neighbouring lung tissue.

There have been occasional reports of patients with aspergilloma who develop progressive symptoms and increasing lung shadows and are found at necropsy to have what is described as aspergillus pneumonia (Leggat and de Kretser, 1968). Perhaps corticosteroids have a place in the management of such patients if this diagnosis could be made with reasonable accuracy.

Recognition of aspergillus pneumonia and pulmonary lesions of the type we have described is important since these patients must be distinguished from those with an aspergilloma with severe and often fatal secondary bacterial infection.

In conclusion, it is evident that when damaged lungs become colonized with aspergilli three patterns of response may be recognized. Growth of fungus may produce a positive precipitin test but no aspergilloma can be demonstrated radiologically. However, surgical excision, necropsy or continued observation may reveal the presence of an apergilloma (Voisin et al., 1964). Often, however, there are no developments and the precipitin test becomes negative, suggesting a transient infection (British Thoracic and Tuberculosis Association, 1970). Alternatively an aspergilloma develops. It may produce no symptoms or it may be associated with an increase in cough or with haemoptysis of varying severity and frequency.
If bleeding is troublesome excision is desirable. Aspergilloma may persist indefinitely but can dis- 음 appear spontaneously or as a result of bacterial $\frac{\omega}{D}$ infection in the cavity. Sometimes an aspergilloma $\stackrel{\odot}{\Omega}$ is associated with severe nonbacterial suppuration in the lung which appears to be mediated by an $\vec{A}$ antigen-antibody reaction. This seems to be an uncommon occurrence but we agree with Hilver- $\vec{\omega}$

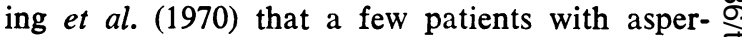
gilloma may benefit from treatment with corti- $\vec{x}$ costeroids without risk of promoting the growth of the fungus.

\section{REFERENCES}

British Thoracic and Tuberculosis Association (1970). Aspergilloma and residual tuberculous cavities-the results of a resurvey. Tubercle (Lond.), 51, 227.

Davies, D. (1970). Lung fibrosis and cavitation mimicking $\overline{\widehat{\rho}}$ tuberculosis. Tubercle (Lond.), 51, 246.

Hilvering, C., Stevens, E. A. M., and Orie, N. G. M. (1970). Fever in aspergillus mycetoma. Thorax, 25, 19.

Kilman, J. W., Ahn, C., Andrews, N. C., and Klassen, K. (1969). Surgery for pulmonary aspergillosis. J. thorac. cardiovasc. Surg., 57, 642.

Leggat, P. O., and de Kretser, D. M. H. (1968). Aspergillus $\bar{O}$ pneumonia in association with an aspergilloma. Brit. J. 气 Dis. Chest, 62, 147.

Longbottom, J. L., and Pepys, J. (1964). Pulmonary aspergillosis: diagnostic and immunological significance $\overrightarrow{\widehat{O}}$ of antigens and C-substance in Aspergillus fumigatus. J. Path. Bact., 88, 141.

Pepys, J. (1969). Hypersensitivity Diseases of the Lungs due to Fungi and Organic Dusts, Monographs in Allergy, Vol. 4, pp. 39-41. Karger, Basle.

Riddle, H. F. V., Channell, S., Blyth, W., Weir, D. M., ฏ) Lloyd, M., Amos, W. M. G., and Grant, I. W. B. (1968). ? Allergic alveolitis in a malt worker. Thorax, 23, 271.

Scadding, J. G. (1967). The bronchi in allergic aspergillosis. Scand. J. resp. Dis., 48, 372.

Stevens, E. A. M., Hilvering, C., and Orie, N. G. M. (1970). Inhalation experiments with extracts of Aspergillus $\mathrm{O}$ fumigatus on patients with allergic aspergillosis and aspergilloma. Thorax, 25, 11.

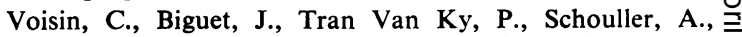
Sergeant, Y. H., and Gernez-Rieux, C. (1964). Asper- $N$ gilloses latentes et tuberculose pulmonaire chronique. o Rev. Tuberc. (Paris), 28, 1311. 